\title{
Implementação de um Detector DTMF utilizando o algoritmo JCO
}

\author{
G. Jerônimo da Silva Jr. e R. M. Campello de Souza
}

\begin{abstract}
Resumo-Este artigo apresenta uma implementação eficiente para um detector DTMF, no sentido de reduzir a complexidade aritmética do procedimento, em relação às técnicas conhecidas. Para isso, a implementação utiliza o novo algoritmo JCO e o algoritmo de Goertzel. Resultados de simulações são apresentados.
\end{abstract}

Palavras-Chave-DTMF, algoritmo JCO, algoritmo de Goertzel, transformada discreta de Fourier, DFT.

Abstract-This paper introduces a new and efficient implementation of a DTMF detector, in the sense that it has low arithmetic complexity. The implementation is based on the new JCO algorithm and in the Goertzel algorithm. Simulation results are presented.

Keywords-DTMF, JCO algorithm, Goertzel algorithm, discrete Fourier transform, DFT.

\section{INTRODUÇÃO}

A transformada de Fourier é uma das ferramentas mais usadas da área de processamento de sinais [1], [2]. Uma função $X(j \Omega)$ é dita ser a transformada de Fourier da função $x(t)$ se

$$
X(j \Omega) \triangleq \int_{-\infty}^{\infty} x(t) e^{-j \Omega t} d t,
$$

onde $j=\sqrt{-1}$. Nesse caso, a função $x(t)$ pode ser escrita em função de $X(j \Omega)$ por

$$
x(t)=\frac{1}{2 \pi} \int_{-\infty}^{\infty} X(j \Omega) e^{j \Omega t} d \Omega .
$$

As equações (1) e (2) são conhecidas como a transformada de Fourier e a transformada inversa de Fourier, respectivamente.

Uma de suas aplicações mais simples é a detecção de uma determinada frequência em um sinal. Com a evolução da eletrônica digital, técnicas baseadas em processamento de tempo discreto tornaram-se atrativas para resolver esse problema. Surgem então a transformada de Fourier de tempo discreto (DTFT, do inglês discrete time Fourier transform), a transformada discreta de Fourier (DFT, do inglês discrete Fourier transform) e algoritmos rápidos para sua computação, as chamadas transformadas rápidas de Fourier (FFT, do inglês fast Fourier transform) [3],[4]. A função $X\left(e^{j \omega}\right)$ é dita ser a transformada de Fourier de tempo discreto da sequência $x[n]$ se

$$
X\left(e^{j \omega}\right) \triangleq \sum_{n=-\infty}^{\infty} x[n] e^{-j \omega n} .
$$

G. J. da Silva Jr. e R. M. Campello de Souza, Grupo de Processamento de Sinais, Departamento de Eletrônica e Sistemas, Universidade Federal de Pernambuco, Recife, PE, E-mails: gilsonjr@gmail.com, ricardo@ufpe.br
A sequência $x[n]$ pode ser escrita como

$$
x[n]=\frac{1}{2 \pi} \int_{-\pi}^{\pi} X\left(e^{j \omega}\right) e^{j \omega n} d \omega .
$$

As equações (3) e (4) são conhecidas como a DTFT e a DTFT inversa, respectivamente. De forma geral, a sequência $x[n]$ é obtida de uma função ou sinal $x(t)$ por $x[n]=x(T n)$. A DFT de uma sequência $x[n]$ de $N$ pontos, $n=0, \ldots, N-1$, denotada por $X[k]$, é dada por

$$
X[k] \triangleq \sum_{n=0}^{N-1} x[n] W_{N}^{k n},
$$

$k=0, \ldots, N-1$, em que $W_{N} \triangleq e^{-j \frac{2 \pi}{N}}$. A sequencia $x[n]$ pode ser escrita como

$$
x[n]=\frac{1}{N} \sum_{k=0}^{N-1} X[k] W_{N}^{-k n} .
$$

As equações (5) e (6) são a DFT e a DFT inversa, respectivamente. A DFT se relaciona com a DTFT pela relação [3]

$$
X[k]=X\left(e^{j \frac{2 \pi}{N} k}\right) .
$$

Existem algoritmos especializados na computação de uma única componente da DFT, dentre eles destacam-se o algoritmo de Goertzel [4]-[6], sliding Goertzel [7],[8] e o JCO [9]. O algoritmo de Goertzel computa a componente $X[k]$ através de uma divisão polinomial pelo polinômio $p_{k}(\chi)$, definido por

$$
\begin{array}{r}
p_{k}(\chi) \triangleq\left(\chi-W_{N}^{k}\right)\left(\chi-W_{N}^{-k}\right) \\
=1-2 \cos \left(\frac{2 \pi k}{N}\right) \chi+\chi^{2} .
\end{array}
$$

O resultado é um algoritmo com $N$ multiplicações reais (supondo $x[n] \in \mathbb{R}$ ) [4]. A Figura 1 mostra a implementação da divisão polinomial por $p_{k}(\chi)$. O algoritmo JCO difere do Goertzel por utilizar o polinômio ciclotômico, $\Phi_{N}(\chi)$, no lugar de $p_{k}(\chi)$. Nesse caso, a divisão polinomial não tem multiplicações, mas são necessárias $2[\phi(L)-1]$ multiplicações finais, onde $\phi($.$) é a função aritmética de Euler e L$ é a ordem de $W_{N}^{k}$ [10],[9]. Esses algoritmos tem como uma de suas principais aplicações a detecção de sinais DTMF (do inglês Dual Tone MultiFrequency). Esses sinais são largamente utilizados em telefonia digital e podem ser reproduzidos, de forma audível, em qualquer aparelho celular. O objetivo é implementar de forma eficiente o detector DTMF, no sentido de reduzir o número de multiplicações e adições do sistema.

O processamento digital de um detector DTMF é mostrado na Figura 2. Nele existem um filtro anti-sobreposição seguido 


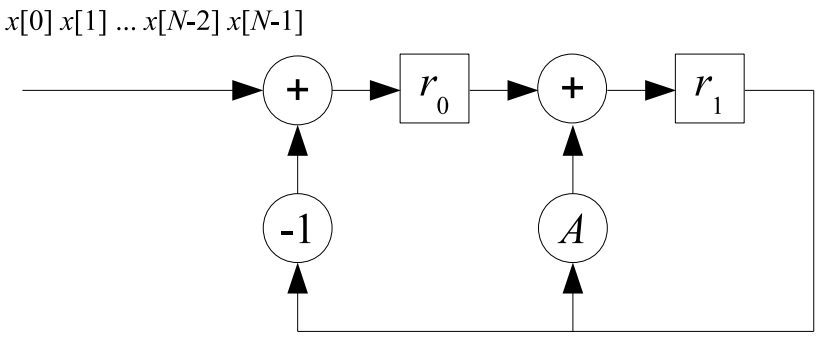

Fig. 1. Filtro autoregressivo para computar a divisão polinomial por $p_{k}(\chi)$. A componente $x[N-1]$ é a primeira a entrar no registrador de deslocamento e $A=2 \cos (2 \pi k / N)$. A componente da DFT é computada por $X[k]=$ $r_{0}+r_{1} W_{N}^{k}$.

de um processamento digital para a detecção do tom DTMF. A Tabela I mostra os caracteres usados em um detector DTMF em função das frequências do sinal. $\mathrm{O}$ tom de um determinado caractere é produzido pelo sinal $v(t)=A\left[\cos \left(2 \pi f_{l} t\right)+\right.$ $\operatorname{sen}\left(2 \pi f_{c} t\right)$ ], onde $f_{l}$ e $f_{c}$ são, respectivamente, as frequências de linha e coluna do caractere.

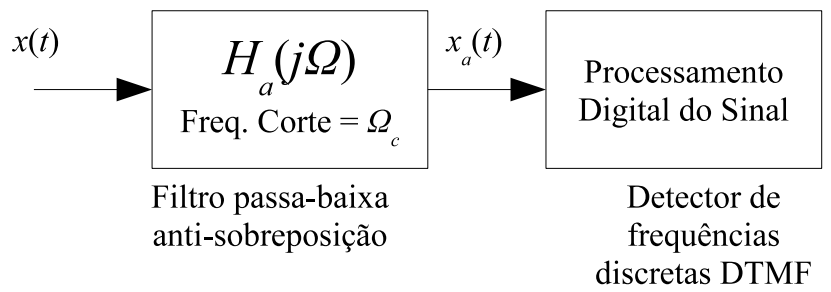

Fig. 2. Diagrama geral de um detector DTMF utilizando processamento de tempo discreto.

TABELA I

CARACTERES DTMF EM FUNÇÃO DAS FREQUÊNCIAS DO SINAL

\begin{tabular}{|c|c|c|c|c|}
\hline$f_{l} \backslash f_{c}(\mathrm{~Hz})$ & 1209 & 1336 & 1477 & 1633 \\
\hline 697 & 1 & 2 & 3 & $\mathrm{~A}$ \\
\hline 770 & 4 & 5 & 6 & $\mathrm{~B}$ \\
\hline 852 & 7 & 8 & 9 & $\mathrm{C}$ \\
\hline 941 & $*$ & 0 & $\#$ & $\mathrm{D}$ \\
\hline
\end{tabular}

Este artigo utiliza a seguinte notação:

$t$ : variável temporal em segundos (s);

$\Omega$ : frequência contínua em rads/s;

$x(t)$ : função ou sinal de entrada;

$X(j \Omega)$ : transformada de Fourier de $x(t)$, definida conforme a relação (1);

$H_{a}(j \Omega)$ : resposta em frequência do filtro passa-baixa de anti-sobreposição;

$\Omega_{c}$ : frequência de corte do filtro anti-sobreposição em rads/s, considerando que $H_{a}(j \Omega)=0$ para $|\Omega| \geq \Omega_{c}$. Também define-se $f_{c} \triangleq \Omega_{c} /(2 \pi)$;

$x_{a}(t)$ : saída do filtro anti-sobreposição;

$X_{a}(j \Omega)$ : transformada de Fourier de $x_{a}(t)$;

$x[n]$ : sequência de $N$ pontos, obtida por $x[n]=x_{a}(T n)$, para $n=0, \ldots, N-1$, sendo $T$ o período de amostragem em segundos;

$X\left(e^{j \omega}\right)$ : DTFT de $x[n]$, definida conforme a relação (3);
$X[k]$ : DFT de $x[n]$, definida conforme a relação (5);

$\tau$ : tempo total de processamento, $\tau \triangleq N T$;

$\Omega_{0}$ : frequência a ser analisada em rads/s, $\Omega_{0}<\Omega_{c}$. Também define-se $f_{0} \triangleq \Omega_{0} /(2 \pi)$.

Este artigo está organizado da seguinte forma: Na Seção II, são apresentadas duas implementações utilizando-se o algoritmo JCO, uma livre de multiplicações e outra com apenas duas multiplicações, para detectar a frequência $f_{0}$ em um sinal de entrada $x(t)$. Na Seção III, demonstra-se como utilizar a implementação da Seção II para construir um detector DTMF completo. Na Seção IV, são mostrados resultados de simulações utilizando-se várias entradas. As conclusões do trabalho são apresentadas na Seção V.

\section{DETECÇÃo DE UMA FREQÜÊNCIA}

O diagrama para detecção de uma frequência está mostrado na Figura 3. A sequência $x[n]$ é obtida através da amostragem de $N$ pontos de $x_{a}(t)$ com período de amostragem

$$
T=\frac{1}{M f_{0}}=\frac{2 \pi}{M \Omega_{0}},
$$

com $M \in \mathbb{N}^{+}$. Ao mesmo tempo da amostragem, o bloco JCO computa uma componente da DFT utilizando o algoritmo JCO.

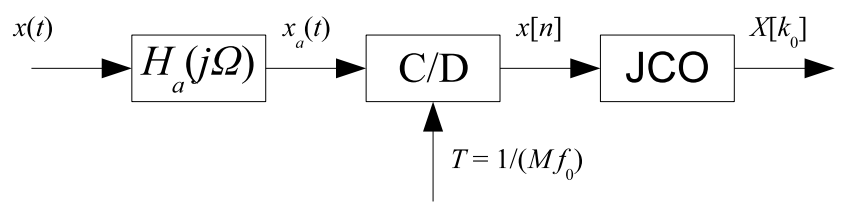

Fig. 3. Diagrama de um processamento digital para detecção de uma frequência. A sequência $x[n]$ é obtida através da amostragem de $N$ pontos de $x_{a}(t)$ com período de amostragem $T=1 /\left(M f_{0}\right)$. A frequência é detectada computando-se uma componente da DFT com a implementação JCO.

A primeira condição que deve ser obedecida é o critério de Nyquist,

$$
M>2 \frac{f_{c}}{f_{0}}=2 \frac{\Omega_{c}}{\Omega_{0}} .
$$

Isso evita o problema de sobreposição da frequência [3], garantindo assim a relação

$$
X\left(e^{j \omega}\right)=\frac{1}{T} X_{a}(j \omega / T) .
$$

Utilizando (7), pode-se escrever

$$
X[k]=\frac{1}{T} X_{a}\left(j \frac{2 \pi}{N T} k\right) .
$$

Considerando (9) e o fato de que $X_{a}(j \Omega)=H_{a}(j \Omega) X(j \Omega)$, tem-se

$$
X\left(j \Omega_{0} \frac{M}{N} k\right)=\frac{2 \pi}{M \Omega_{0} H_{a}\left(j \Omega_{0} \frac{M}{N} k\right)} X[k] .
$$

Agora, escolhe-se $N$ múltiplo de $M$, isto é,

$$
N=k_{0} M,
$$

onde $k_{0} \in \mathbb{N}^{+}$. Fazendo $k=k_{0}$ na Equação (13), tem-se

$$
X\left(j \Omega_{0}\right)=\frac{2 \pi}{M \Omega_{0} H_{a}\left(j \Omega_{0}\right)} X\left[k_{0}\right] .
$$


A frequência $\Omega_{0}$ pode ser detectada computando-se a componente $k_{0}$ de uma DFT de $N$ pontos. Agora, pode-se projetar o filtro digital que implementa o algoritmo JCO.

A computação da componente $X\left[k_{0}\right]$, pode ser feita utilizando o filtro IIR

$$
H(z)=\frac{1}{1-W_{N}^{-k_{0}} z^{-1}},
$$

com entrada $x[n], n=0, \ldots, N-1$ e saída $y[n]$ [3],[6]. A componente de interesse é computada por

$$
X\left[k_{0}\right]=y[N] .
$$

A implementação do algoritmo JCO é feita escrevendo-se $H(z)$ como

$$
H(z)=H(z) \frac{\prod_{\operatorname{ord}\left(W_{N}^{i}\right)=L, i \neq k_{0}}\left(1-W_{N}^{-i} z^{-1}\right)}{\prod_{\operatorname{ord}\left(W_{N}^{i}\right)=L, i \neq k_{0}}\left(1-W_{N}^{-i} z^{-1}\right)},
$$

que pela definição de polinômio ciclotômico [4],[11], torna-se

$$
H(z)=\frac{\prod_{\operatorname{ord}\left(W_{N}^{i}\right)=L, i \neq k_{0}}\left(1-W_{N}^{-i} z^{-1}\right)}{\Phi_{L}\left(z^{-1}\right)} .
$$

Os graus do denominador e do numerador são $\phi(L)$ e $\phi(L)-$ 1 , respectivamente. Dessa forma, $H(z)$ pode ser expresso na forma

$$
H(z)=\frac{1+a_{1} z^{-1}+\ldots+a_{\phi(L)-1} z^{-\phi(L)+1}}{1+b_{1} z^{-1}+\ldots+z^{-\phi(L)}} .
$$

A Figura 4 mostra a implementação em hardware do algoritmo JCO no caso geral.

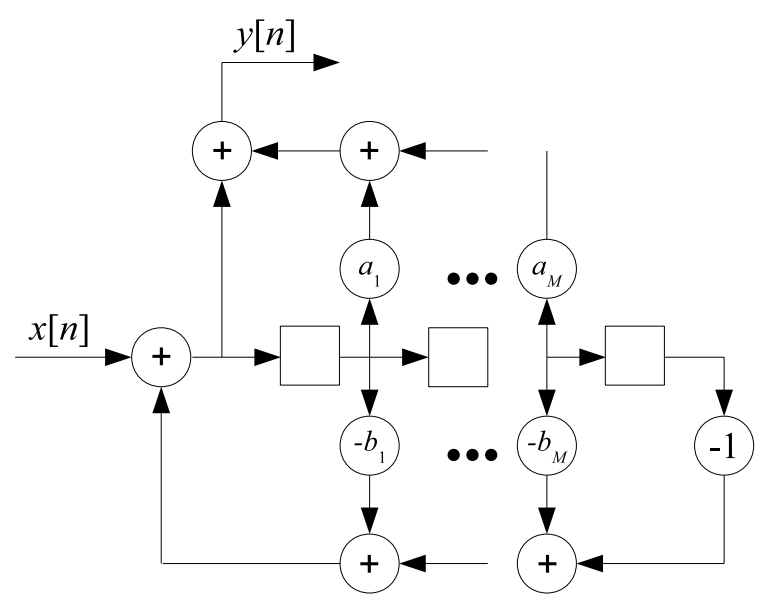

Fig. 4. Diagrama do filtro que implementa o algoritmo JCO. A componente da DFT é computada por $X\left[k_{0}\right]=y[N]$.

A complexidade multiplicativa do JCO é proporcional a

$$
L=\phi\left(\frac{N}{\operatorname{MDC}\left(N, k_{0}\right)}\right),
$$

e $k_{0}$ divide $N$, pois $N / k_{0}=M \in \mathbb{N}^{+}$. Nesse caso, a Equação (21) torna-se

$$
L=\phi(M) .
$$

Quanto menor o valor de $M$, menor a complexidade do procedimento. Entretanto, o critério de Nyquist apresentado na Inequação (10) impõe uma restrição no projeto. Filtros com $\Omega_{0}$ próximo a $\Omega_{c}$ são mais difíceis de implementar, o que implica que diminuir a complexidade do algoritmo JCO significa aumentar a complexidade do filtro anti-sobreposição. Tipicamente, o filtro anti-sobreposição é um filtro Butterworth de primeira ordem, implementado com capacitores e resistores [12]. É necessário, portanto, escolher de forma adequada o parâmetro $M$, de acordo com o projeto. A seguir, são apresentadas duas escolhas eficientes para o parâmetro $M$.

\section{A. Implementação JCO com $M=4$}

Nesse caso, o filtro digital que implementa o JCO, utilizando (19), é

$$
H(z)=\frac{1+j z^{-1}}{\Phi_{4}\left(z^{-1}\right)}=\frac{1+j z^{-1}}{1+z^{-2}}
$$

que coincide com o filtro encontrado utilizando-se o algoritmo de Goertzel. A implementação está mostrada na Figura 5. Este projeto computa a componente $X\left[k_{0}\right]$ utilizando apenas $N$ adições com a restrição de que $\Omega_{0}<\Omega_{c}<2 \Omega_{0}$.

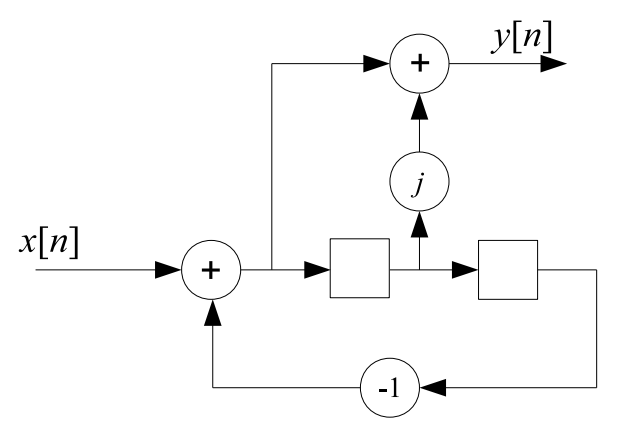

Fig. 5. Implementação do algoritmo JCO com $M=4$. Existem apenas multiplicações triviais.

\section{B. Implementação JCO com $M=8$}

Nesse caso, o filtro JCO é

$$
H(z)=\frac{\left(1-W_{8} z^{-1}\right)\left(1-W_{8}^{3} z^{-1}\right)\left(1-W_{8}^{5} z^{-1}\right)}{\Phi_{8}\left(z^{-1}\right)},
$$

que leva a

$$
H(z)=\frac{1+\frac{\sqrt{2}}{2}(1+j) z^{-1}+j z^{-2}-\frac{\sqrt{2}}{2}(1-j) z^{-3}}{1+z^{-4}} .
$$

A implementação desse filtro é mostrada na Figura 6. Esse projeto utilizando o algoritmo JCO computa a componente $X\left[k_{0}\right]$ utilizando apenas duas multiplicações e aproximadamente $N$ adições, o que é muito mais eficiente do que o algoritmo de Goertzel, que computa a mesma componente utilizando aproximadamente $N$ multiplicações e $2 N$ adições. A restrição para a frequência de corte do filtro anti-sobreposição, neste caso, é $\Omega_{0}<\Omega_{c}<4 \Omega_{0}$. 


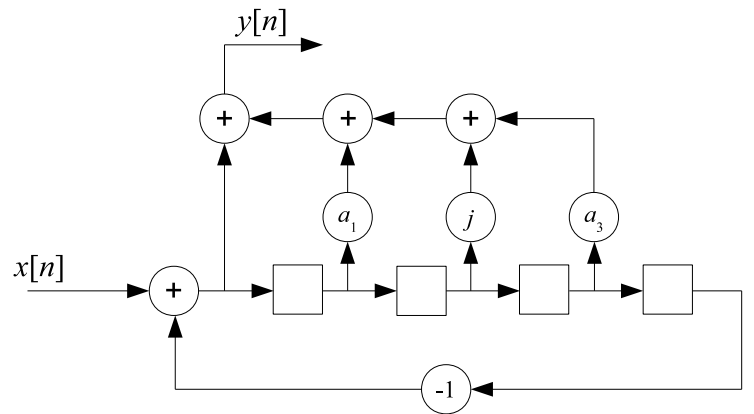

Fig. 6. Implementação do algoritmo JCO com $M=8, a_{1}=\frac{\sqrt{2}}{2}(1+j) \mathrm{e}$ $a_{3}=\frac{\sqrt{2}}{2}(-1+j)=-a_{1}^{*}$.

\section{DETECTOR DTMF}

O detector DTMF é implementado de acordo com a Figura 7. Como é utilizado apenas um filtro anti-sobreposição, nem todos os filtros JCO podem ser tipo $M=4$ (observando as restrições para todos). Outra observação é que os tempos de computação $\tau$ devem ser compatíveis, de modo que o processamento de todas as frequências termine simultaneamente; para isso basta escolher $k_{i} \approx \tau f_{i}$ com $k_{i} \in \mathbb{N}^{+}$. Assim é necessário escolher o número de pontos $N_{i}$ para cada frequência $f_{i}$ analisada.

Adotando $\tau=40 \mathrm{~ms}$, que é um valor encontrado nos circuitos integrados comerciais, temos a Tabela II com os parâmetros do projeto do detector DTMF para $f_{c}=2,4 k H z$. A frequência de corte do filtro anti-sobreposição deve satisfazer a condição de projeto para todas as frequências.

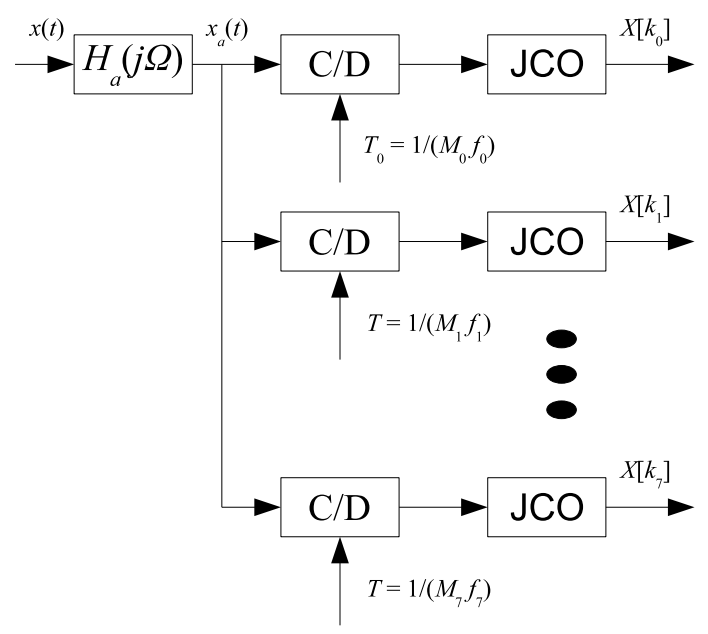

Fig. 7. Implementação do detector DTMF utilizando o algoritmo JCO.

O projeto detecta todas as frequências DTMF num sinal $x(t)$ com duração de $40 \mathrm{~ms}$ com aproximadamente 10 multiplicações, 2130 adições e 26 registradores utilizados pelos filtros JCO (5 filtros de ordem 4 e 3 filtros de ordem 2). As frequências são detectadas utilizando-se a Equação (15), que pode ser reescrita por

$$
X\left(j \Omega_{i}\right)=\frac{2 \pi}{M_{i} \Omega_{i} H_{a}\left(j \Omega_{i}\right)} X\left[k_{i}\right] .
$$

TABELA II

PARÂMETROS DE PROJETO DO DETECTOR DTMF IMPLEMENTADO UTILIZANDO-SE O ALGORITMO JCO: A FREQUÊNCIA ANALISADA $f_{i}$, O ÍNDICE DA COMPONENTE DFT $k_{i} \approx f_{i} \tau$, O TEMPO DE COMPUTAÇÃO $\tau_{i}=k_{i} / f_{i}$, O PARÂMETRO $M_{i}$ E O NÚMERO DE PONTOS AMOSTRADOS POR COMPUTAÇÃO $N_{i}=M_{i} k_{i}$, ASSUMINDO $f_{c}=2,4 k H z$.

\begin{tabular}{|c|c|c|c|c|}
\hline$f_{i}(\mathrm{~Hz})$ & $k_{i}$ & $\tau_{i}(\mathrm{~ms})$ & $M_{i}$ & $N_{i}$ \\
\hline 697 & 27 & 38,7 & 8 & 216 \\
\hline 770 & 31 & 40,3 & 8 & 248 \\
\hline 852 & 34 & 39,9 & 8 & 272 \\
\hline 941 & 38 & 40,4 & 8 & 304 \\
\hline 1209 & 48 & 39,7 & 8 & 384 \\
\hline 1336 & 53 & 39,7 & $4 / 8$ & $212 / 424$ \\
\hline 1477 & 59 & 39,9 & $4 / 8$ & $236 / 472$ \\
\hline 1633 & 65 & 39,8 & $4 / 8$ & $260 / 520$ \\
\hline
\end{tabular}

A Tabela III apresenta uma comparação da complexidade aritmética do projeto entre os algoritmos JCO e Goertzel.

\section{TABELA III}

COMPARAÇÃO DA COMPLEXIDADE ARITMÉTICA DO PROJETO DTMF UTILIZANDO OS ALGORITMOS JCO E GOERTZEL. AS MULTIPLICAÇÕES CONTABILIZADAS SÃO TODAS NÃO TRIVIAIS. CONSIDERA-SE QUE $x(t) \in \mathbb{R}$ E $f_{c}=2,4 k H z$.

\begin{tabular}{|c|c|c|}
\hline Complexidade & Goertzel & JCO \\
\hline Adições & 3556 & 2131 \\
\hline Multiplicações & 1424 & 10 \\
\hline Registradores & 16 & 26 \\
\hline
\end{tabular}

\section{SimulaçÃo e Resultados}

Foram feitos dois tipos de simulação, um com sinais sintetizados, gerados digitalmente, e outro com sinais obtidos através de um microfone, sujeito a ruído e interferência. No primeiro teste, não há necessidade de se utilizar um filtro antisobreposição. No segundo teste, foi utilizado um filtro digital para simular o filtro anti-sobreposição. A seguir, são descritos alguns detalhes dos dois testes.

\section{A. Teste com Sinais Sintetizados}

Neste teste, um sinal de entrada $x(t)$ foi gerado pela relação

$$
x(t)=\cos (2 \pi 697 t)+\operatorname{sen}(2 \pi 1477 t) .
$$

O sinal $x(t)$ foi inserido na estrutura da Figura $7 \mathrm{com}$ $H_{a}(j \Omega)=1$. As frequências foram computadas por (26). O resultado é apresentado no gráfico da Figura 8 , em que é possível detectar claramente as duas frequências do sinal.

\section{B. Teste com Sinais Amostrados de Ambiente com Ruído}

Neste teste, foi utilizada uma sala com condicionador de ar e ventilador ligado e um celular para reproduzir o som da tecla $5\left(f_{l}=770 \mathrm{~Hz}\right.$ e $\left.f_{c}=1336 \mathrm{~Hz}\right)$ em um microfone ligado a um computador pessoal. $O$ sinal foi amostrado a uma taxa de $44100 \mathrm{~Hz}$. Após o sinal ser amostrado, o filtro anti-sobreposição foi aplicado de forma digital, isto é, o filtro $H_{a}(j \Omega)$ é simulado. O ideal seria se utilizar um filtro analógico antes da amostragem. O filtro simulado escolhido foi 


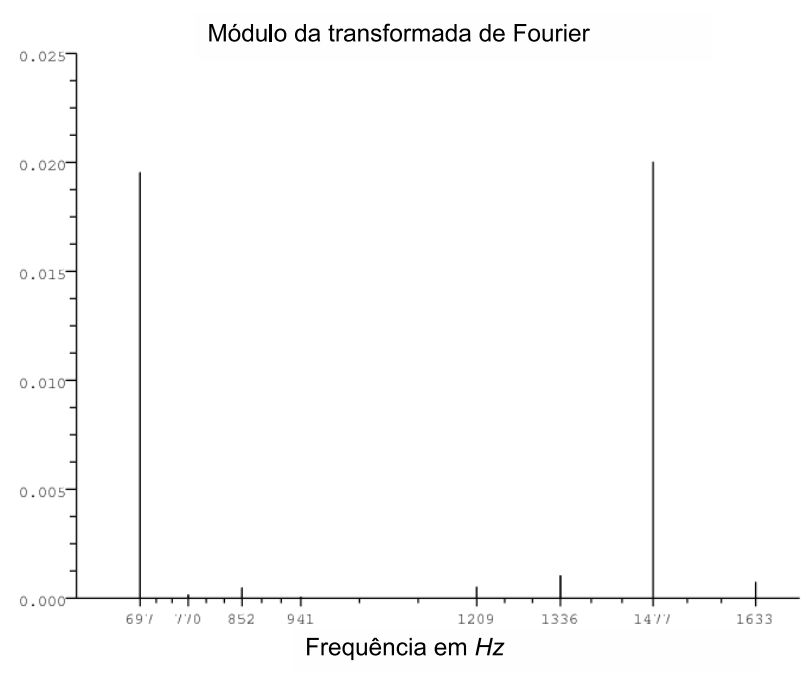

Fig. 8. Resultado do teste com o sinal sintetizado $x(t)=\cos (2 \pi 697 t)+$ $\operatorname{sen}(2 \pi 1477 t)$ e $H_{a}(j \Omega)=1$. O gráfico mostra a magnitude da transformada de Fourier, computada por (26), em função da frequência $f_{i}$.

um filtro de Butterworth de primeira ordem com frequência de corte $f_{c}=2,4 k H z$, isto é,

$$
H_{a}(j \Omega)=\frac{2 \pi f_{c}}{2 \pi f_{c}+j \Omega} .
$$

O resultado dessa simulação está mostrado na Figura 9. Mesmo sem implementar o filtro anti-sobreposição, é possível detectar as duas frequências que caracterizam a tecla 5 do DTMF.

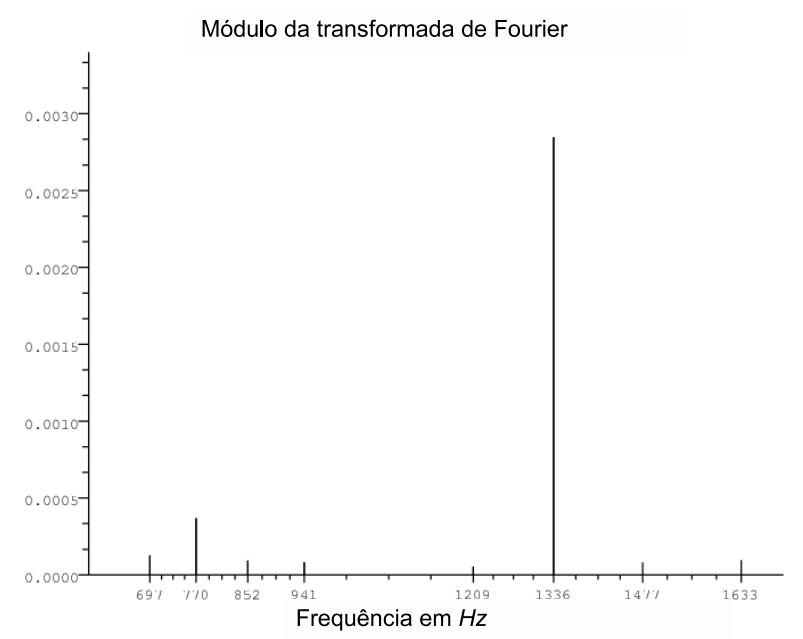

Fig. 9. Resultado do teste com o sinal obtido de um ambiente com ruído. O gráfico mostra a magnitude da transformada de Fourier, estimada pela Equação (26), em função da frequência $f_{i}$.

\section{Conclusões}

Esse artigo apresentou uma maneira eficiente de se implementar uma detecção de frequência de um sinal analógico utilizando o algoritmo JCO, que para determinadas situações torna-se igual ao algoritmo de Goertzel. Também foi projetado um detector DTMF com essa implementação e simulações foram realizadas para demonstrar a validade da estrutura no processamento de sinais analógicos.

O critério usado para se caracterizar a eficiência do algoritmo foi o de reduzir o número de multiplicações e adições dos filtros digitais. Isso é importante para diminuir a complexidade do hardware, diminuir o número de operações por segundo e, consequentemente, diminuir a energia consumida em um processamento.

A detecção de sinais DTMF pode ser feita de outras maneiras [13],[14], aparentemente, não mais eficientes do que a apresentada nesse trabalho.

Embora este artigo mostre uma aplicação para detecção de sinais DTMF, é possível utilizar o algoritmo JCO em muitas outras aplicações, tais como análise espectral, filtragem de frequências específicas, estimação de resposta em frequência e equalização, entre outras.

\section{AGRADECIMENTOS}

Os autores agradecem ao Prof. Dr. Hélio M. de Oliveira por suas valorosas contribuições ao presente trabalho. $\mathrm{O}$ primeiro autor agradece ao $\mathrm{CNPq}$ pelo apoio recebido durante a realização desse trabalho.

\section{REFERÊNCIAS}

[1] A. V. Oppenheim, A. S. Willsky, and S. H. Nawab, Signals and Systems, 2nd ed. Prentice Hall, 1996.

[2] H. M. de Oliveira, Análise de Fourier e Wavelets: sinais estacionários e não estacionários. Univesitária da UFPE, 2007.

[3] A. V. Oppenheim, R. W. Schafer, and J. R. Buck, Discrete-Time Signal Processing, 2nd ed. Prentice Hall, 1999.

[4] R. E. Blahut, Fast Algorithms for Digital Signal Processing. AddisonWesley Publishing Company, 1984.

[5] G. Goertzel, "An algorithm for the evaluation of finite trigonometric series," The American Mathematical Monthly, vol. 65, no. 1, pp. 34-35, 1958.

[6] J. Beraldin and W. Steenaart, "Overflow analysis of a fixed-point implementation of the Goertzel algorithm," Circuits and Systems, IEEE Transactions on, vol. 36, no. 2, pp. 322-324, Feb 1989.

[7] J. F. Chicharo and M. T. Kilani, "A sliding Goertzel algorithm," Signal Processing, vol. 52, no. 3, pp. 283 - 297, 1996.

[8] R. Garcia-Retegui, S. Gonzalez, M. Funes, and S. Maestri, "Implementation of a novel synchronization method using sliding Goertzel DFT," Intelligent Signal Processing, 2007. WISP 2007. IEEE International Symposium on, pp. 1-5, Oct. 2007.

[9] G. J. da Silva Jr., R. M. C. de Souza, and H. M. de Oliveira, "New algorithms for computing a single component of the discrete Fourier transform," 10th International Symposium on Communication Theory and Application, ISCTA'09, july 2009, aceito para apresentação.

[10] D. M. Burton, Elementary Number Theory, 6th ed. McGraw-Hill, 2007.

[11] R. J. McEliece, Finite Fields for Computer Scientists and Engineers. Kluwer Academic Publishers, 1987.

[12] A. S. Sedra and K. C. Smith, Microeletrônica, 4th ed. MAKRON Books, 2000.

[13] R. Beck, A. Dempster, and I. Kale, "Finite-precision Goertzel filters used for signal tone detection," Circuits and Systems II: Analog and Digital Signal Processing, IEEE Transactions on, vol. 48, no. 7, pp. 691-700, Jul 2001.

[14] V. Vassilevsky, "Efficient multitone detection [dsp tips \& tricks]," Signal Processing Magazine, IEEE, vol. 24, no. 2, pp. 144-147, March 2007. 